\section{Kortisonangst: In der pneumologischen Rehabilitation geringer als erwartet. Ergebnisse einer Patientenbefragung ${ }^{1}$}

Zusammenfassung: Hintergrund: Kortikoide, systemisch oder lokal appliziert, sind in der Behandlung von Erkrankungen des oberen und unteren Atemwegstraktes nicht mehr wegzudenken. In der Literatur finden sich Hinweise, dass bei vielen Patienten Vorbehalte gegenüber einer derartigen Medikation bestehen, die teilweise so stark sind, dass von einer regelrechten Kortisonangst gesprochen wird. Schlechte Compliance und damit häufig eine Verschlechterung des Krankheitsbildes sind vorprogrammierte Folgen. Ziel der Arbeit war die Untersuchung, wie ausgeprägt die Angst vor Kortison bei Patienten in der pneumologischen Rehabilitation ist und ob Beziehungen zu anderen Einstellungsbereichen bestehen. Methode: Patienten mit COPD und Asthma mit inhalativer und/oder systemischer Kortikoid-Medikation erhielten während ihres vierwöchigen stationären Reha-Aufenthaltes einen umfangreichen Fragebogen. In die Auswertung gelangten 102 Fragebogen. Ergebnisse: Die Angaben der Patienten sprachen für eine hohe Compliance bez. der Kortikoidmedikation. Was die Angst vor derartigen Präparaten betrifft, so lieferten lediglich 5/102 (4,9\%) der Befragten Werte, aus denen auf eine stark ausgeprägte Kortisonangst geschlossen werden kann. Kortisonangst steht $u$.a. in einem mäßigen Zusammenhang zu allgemeiner psychischer Belastung und einer allgemein negativen Einstellung gegenüber Medikamenten. Compliance bei der Medikamenteneinnahme korreliert negativ mit Kortisonangst. Schlussfolgerungen: Entgegen Angaben in der Literatur von weitverbreiteten Vorbehalten und Ängsten gegenüber Kortison spielen diese zumindest bei dem meist älteren Klientel pneumologischer Rehabilitation nach unseren Befunden eine eher geringe Rolle. Erklärungsmöglichkeiten werden diskutiert.

Fear of Cortison: In Pulmonary Rehabilitation less than expected. Results of a Patient's Questionnaire: Background: The treatment of diseases of the upper and lower respiratory tracts can't be imagined without corticoids, applied systemically of locally. In specialist literature one can find indications of a certain reservation towards this kind of medication on the part of many patients. Sometimes, this reservation is so strong that it can be referred to as a regular fear of cortison. Bad compliance and with it, frequently, a deterioration of the symptoms, are inevitable consequences. The aim of the study was to find out how distinct the fear of cortison is with patients in pneumological rehabilitation and wether there are any relations with other spheres of attitude. Method: Patients with

Pneumologie 2000; 54: 68-71

(C) Georg Thieme Verlag Stuttgart · New York ISSN 0934-8387

\author{
A. Hirschbichler, S. Spohn, W. Petro \\ Klinik Bad Reichenhall der LVA Ndb.-Opf. \\ Fachklinik für Erkrankungen der Atmungsorgane und Allergien, \\ Bad Reichenhall (Chefarzt: Prof. Dr. W. Petro)
}

COPD and asthma with inhalative and/or systemic corticoid medication received an extensive questionnaire during their rehabilitation as in-patients for four weeks. 102 questionnaires were evaluated. Results: The statements of the patients indicated high compliance regarding corticoid medication. As to the fear of such preparation, only $5 / 102(4,9 \%)$ of those questioned produced results which suggest strong fear of cortison. Fear of cortison is, among other things, slightly related to general psychic stress and a general negative attitude towards medicine. There is a negative correlation between compliance at the taking of medicine and fear of cortison. Conclusions: Contrary to statements in specialist literature of wide-spread reservations and fear towards cortison, we have found out that these play only a minor part, at least with the mostly elderly clientele of pneumological rehabilitation. Different explanations are discussed.

\section{Einleitung}

Die Vorstellungen zur Pathogenese des Asthma bronchiale haben in den letzten Jahren einen grundlegenden Wandel erfahren, der nicht nur von theoretischem Interesse ist, sondern auch erhebliche praktische therapeutische Implikationen zur Folge hat. Im Zentrum des Interesses steht die bronchiale Hyperreaktivität als Kernsymptom der Asthmakrankheit.

Als therapeutisches Hauptziel wird heute nicht nur die Verringerung oder Beseitigung der Bronchialobstruktion, sondern die Unterdrückung der bronchialen Hyperreaktivität angesehen. Damit wandelt sich das gesamte Konzept der Asthmatherapie: Während Glukokortikoide noch vor einigen Jahren am Ende der therapeutischen Möglichkeiten eingestuft wurden, stehen sie heute in inhalativer Form am Anfang der Asthmatherapie. Weitere Indikationen bestehen nach heutigem Konsens bei chronischer Bronchitis mit Hyperreagibilität, Obstruktion oder Emphysemkomponente. Kortikoide, systemisch oder lokal appliziert, sind in der Behandlung von Erkrankungen des oberen und unteren Atemwegstraktes nicht mehr wegzudenken $[1,2,3]$.

Dennoch stellt die Verordnung von Kortikoiden, auch von lokal wirksamen, oft ein Problem dar. Nach wie vor haben viele Patienten Vorbehalte gegenüber einer derartigen Medi-

\footnotetext{
1 Die Arbeit wurde in Kurzform als Poster beim DGP-Kongress in Bad Reichenhall im März 1999 (18. bis 20. 3. 1999) vorgestellt.
} 
kation, teilweise so stark, dass von einer regelrechten Kortison-Angst gesprochen wird [4]. Diese für den behandelnden Arzt kaum nachvollziehbare Angst kann auch durch wiederholte Sachinformationen oft nur unzureichend überwunden werden $[5,6,7,8]$. Schlechte Compliance und damit häufig eine Verschlechterung des Krankheitsbildes sind vorprogrammierte Folgen. Gerade bei „Risikopatienten“, d.h. Patienten, die immer wieder mit schwersten lebensbedrohlichen Asthmaanfällen behandelt werden müssen, scheint häufig mangelhafte Compliance, gerade was die Kortisonmedikation betrifft, vorzuliegen [9,10]. Die Ursachen und Hintergründe dieser Vorbehalte und Ängste sind komplex [11,12,13,14].

Wegen der Vielzahl bedingender, kaum kontrollierbarer Variablen erweist sich empirische Forschung in diesem Bereich grundsätzlich als problematisch. Diese Feststellung betrifft die gesamte Compliance-Forschung (z.B. [15]).

Es war das Ziel dieser Arbeit, zu untersuchen:

- wie ausgeprägt die Angst vor Kortison bei Patienten in der stationären pneumologischen Rehabilitation ist sowie

- ob und inwieweit Beziehungen zwischen der Angst vor Kortison und compliance-relevanten Einstellungen der Patienten bestehen. Einstellungen werden in der Psychologie als relativ dauerhafte, sowohl die Wahrnehmung als auch das Verhalten bestimmende individuelle Überzeugungen definiert.

\section{Patienten und Methode}

In der explorativen Studie untersucht wurden Patienten mit COPD und/oder Asthma mit inhalativer und/oder systemischer Kortikoidtherapie in der stationären pneumologischen Rehabilitation. Im Erhebungszeitraum von sechs Monaten erhielten 165 konsekutiv aufgenommene Patienten zu Beginn ihres vierwöchigen Reha-Aufenthaltes in der Fachklinik Bad Reichenhall einen umfangreichen Fragebogen nach Deuchert \& Petermann [11]. Die Rücklaufquote lag bei 131/165 (79,4\%). Die Stichprobe ist als repräsentativ für kortikoidpflichtige Patienten der Klinik anzusehen.

Von den befragten Patienten waren 52 (40\%) weiblich und 79 (60\%) männlich.

Die Patienten waren zwischen 23 und 81 Jahre alt, das Durchschnittsalter lag bei 54,5 Jahren. Zum Zeitpunkt der Befragung arbeitsfähig erwiesen sich 43,5\%, 29,8\% waren berentet, 13,7\% arbeitsunfähig, 8,1\% arbeitslos, $4 \%$ erwerbsunfähig und $0,8 \%$ berufsunfähig.

Die Dauer der Erkrankung schwankte zwischen 3 Monaten und 52 Jahren. Der Mittelwert lag bei 13 Jahren.
Von den Befragten nahmen 87,8\% Kortisonpräparate ein. Kortisontabletten wurden von $45,8 \%$ der Patienten eingenommen, während 93,9\% Kortison inhalierten.

11 von 131 Patienten (8,3\%) füllten den Fragebogen völlig korrekt aus. 29/131 der Fragebogen (22\%) wurden wegen $\mathrm{zu}$ vieler fehlender Angaben (missing values > 10\%) nicht ausgewertet. In die Auswertung gelangten 102 der 131 Fragebogen.

\section{Ergebnisse}

\section{Informationsinteresse}

$72 \%$ der Patienten gaben an, sich sehr stark über Atemwegserkrankungen $\mathrm{zu}$ informieren, $16,8 \%$ hatten ein starkes Interesse. Bei der Frage nach eingeholten Informationen über Kortison ergab sich ein ähnliches Bild: 70,5\% gaben ein sehr starkes Interesse, $17,2 \%$ ein starkes Interesse an.

\section{Umgang mit Kortison}

Im Umgang mit Kortison entsprachen die Angaben der Patienten weitgehend den Erwartungen an eine effektive Kortisontherapie.

Demzufolge hielten 93\% der Patienten die Aussage „Kortison nehme ich trotz ärztlicher Verordnung nie“ für gar nicht zutreffend. Die Patienten achteten nach ihren Angaben stark darauf, ihre Kortisonpräparate immer zu den richtigen Zeitpunkten einzunehmen.

Von 79\% der Patienten wurde die Aussage „Ich setze das Kortison immer vorübergehend $a b$, wenn ich gerade Berichte über die Nebenwirkungen gehört habe“ als gar nicht zutreffend zurückgewiesen. Die Patienten hielten die Aussage „Ich nehme Kortison, wie ich will“ zu 93\% für gar nicht zutreffend, scheinen also die Verordnungen des Arztes strikt zu befolgen. $34 \%$ gaben an, Kortison nur einzunehmen, wenn es ihnen schlecht gehe, die Mehrzahl (54\%) wies diese Aussage als gar nicht zutreffend zurück.

\section{Statistische Maße der Aussagenbereiche}

Die Werte der internen Konsistenz (Cronbach's Alpha) als Determinanten der Messzuverlässigkeit fallen zum Teil geringer aus als in den Berechnungen von Deuchert \& Petermann [11]. Die vorliegenden Ergebnisse sprechen bei vier von sieben Subskalen eher dafür, dass jeweils Einstellungen zu unterschiedlichen Aspekten gemessen wurden (Variable 2, 3, 4 und 6) (Tab. 1).

\begin{tabular}{llll}
\hline Variable & Items & Cronbach's Alpha & Missing Values \\
\hline 1. psychosoziale Belastungen & 29 &, 8798 & $3,84 \%$ \\
2. Einstellung zu Medikamenten & 8 &, 4432 & $2,58 \%$ \\
3. Compliance bei Medikamenteneinnahme & 6 &, 2628 & $1,15 \%$ \\
4. Kortisonangst & 7 &, 4982 & $7,56 \%$ \\
5. Kortison-Compliance & 6 &, 5876 & $5,60 \%$ \\
6. soziale Unterstützung & 13 &, 4710 & $4,82 \%$ \\
7. Arztbild & 12 &, 5738 & $1,59 \%$ \\
\hline
\end{tabular}

Tab. 1 Aussagenbereiche/Variablen und innerer Zusammenhang; Alpha-Koeffizient von Cronbach als Maß der internen Konsistenz der Einstellungsbereiche 
Tab. 2 Items zur Erfassung von Kortisonangst

- Kortison vergiftet den Körper

- Kortison sollte am besten nie eingenommen werden

- Ich bin dankbar, dass es Kortison gibt

- Kortison bringt den Körper aus dem inneren Gleichgewicht

- Die Nebenwirkungen des Kortison sind schlimmer als die eigentliche Krankheit

- Kortison ist ein Teufelszeug

- Kortison lindert die schlimmsten Krankheitsfolgen

Da Kortisonangst die zentrale Variable der Untersuchung war, sollen die diesbezüglichen Items nach Deuchert \& Petermann [11] im Folgenden angeführt werden (Tab. 2).

Die Skalierung der Antworten erfolgte auf einer fünf-stufigen Ratingskala zwischen (1) „trifft gar nicht zu“ und (5) „trifft voll zu“.

\section{Häufigkeit von Kortisonangst}

Die Auswertung der Fragebogen ergibt, dass nur bei 5/102 $(=4,9 \%)$ der Patienten von einer ausgesprochenen Kortisonangst gesprochen werden kann. Immerhin 16/102 (=15,6\%) der befragten Patienten liefern Werte, aus denen auf eine mittlere Ablehnung von Kortisonpräparaten zu schließen ist.

Somit liefern 21/102 (=20,5\%) der Patienten Zahlenwerte, aus denen auf eine starke bis mittlere Ablehnung von Kortisonpräparaten geschlossen werden kann (Abb. 1, Tab. 3).

- Kortisonangst steht mit allgemeiner psychischer Belastung im Zusammenhang $(\mathrm{r}=-.32, \mathrm{p}<.005)$.

- Kortisonangst steht mit einer allgemein negativen Einstellung gegenüber Medikamenten im Zusammenhang $(\mathrm{r}=-$ $.32, \mathrm{p}<.005)$.

- Compliance bei der Medikamenteneinnahme steht im Zusammenhang mit einer allgemein positiven Einstellung Medikamenten gegenüber $(\mathrm{r}=.29, \mathrm{p}<.005)$ und korreliert negativ mit Kortisonangst $(\mathrm{r}=-.51, \mathrm{p}<.005)$.

- eine niedrige Compliance bei der Einnahme von Kortisonpräparaten steht im Zusammenhang mit stark ausgeprägter Kortisonangst $(\mathrm{r}=-.26, \mathrm{p}<.005)$.

- eine allgemein ablehnende Haltung Medikamenten gegenüber steht mit einer schlechten Kortison-Compliance im Zusammenhang $(\mathrm{r}=.39, \mathrm{p}<.005)$.

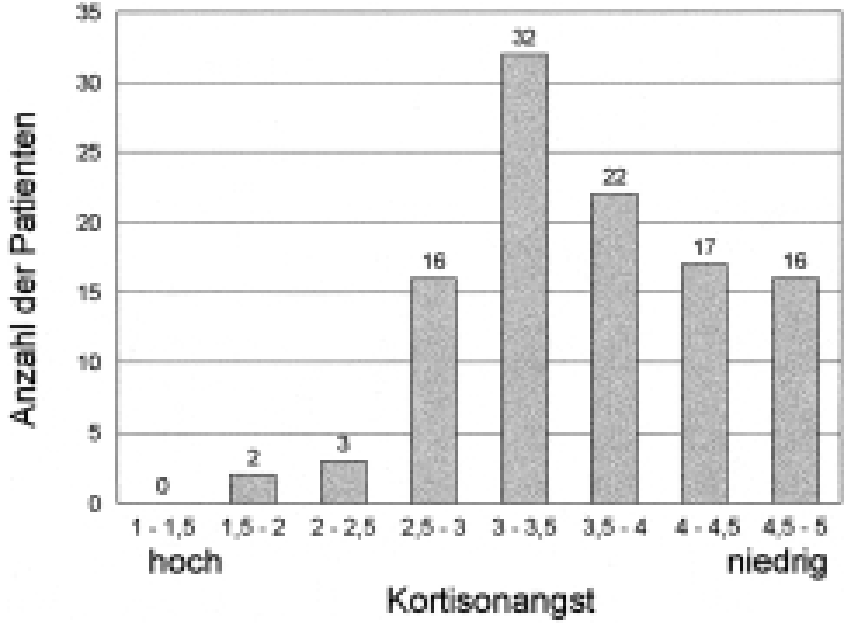

Abb. 1 Kortisonangst: Werte der Patienten $(n=102)$ auf Fragen bezüglich Ablehnung von Kortisonpräparaten (geringe Werte $=$ stark ausgeprägte Kortisonangst)

- die Compliance bei der Einnahme speziell von Kortisonpräparaten steht im Zusammenhang mit der Compliance, was Medikamente allgemein betrifft $(\mathrm{r}=.33, \mathrm{p}<.005)$.

- Arztbild und Einstellung Medikamenten gegenüber stehen in einem negativen Zusammenhang $(r=-.27, p<.05)$, ebenso Arztbild und Kortisoncompliance $(r=-.20, p<.05)$. D. h.: eine positive Einschätzung des Arztes hängt mit einer ablehnenden Haltung gegenüber Medikamenten allgemein und niedriger Compliance, was Kortisonpräparate betrifft, zusammen.

\section{Diskussion}

Die Patientenangaben bez. ihres Informationsinteresses über Kortison als auch ihrer Bereitschaft, verordnete Präparate genau nach ärztlicher Vorgabe einzunehmen, legen eine sehr hohe Compliance nahe und sprechen im Weiteren dafür, dass Kortisonangst in der stationären pneumologischen Rehabilitation eine eher untergeordnete Rolle spielt.

Die Ergebnisse bestätigen im Wesentlichen die von Deuchert \& Petermann, die bei höchstens $7 / 53$ (=13,2\%) vergleichbaren Patienten Werte fanden, aus denen auf starke bis mittlere Ängste vor Kortisonpräparaten zu schließen war [11]. Nachgewiesen wurde von den Autoren auch interessanterweise, dass

Tab. 3 Zusammenhänge zwischen Kortisonangst und anderen Einstellungsbereichen

\begin{tabular}{llllll}
\hline & Psych. Belast. & Kort. Ang. & Einst. Med. & Compl. Med. & Compl. Kort. \\
\hline Kort. Ang. & $-0,32^{* *}$ & & & & soz. Unterst. \\
Einst. Med. & $0,32^{* *}$ & $-0,51^{* *}$ & & & \\
Compl. Med. & 0,21 & $-0,26^{* *}$ & $0,29^{* *}$ & & \\
Compl. Kort. & 0,17 & $-0,03$ & $0,39^{* *}$ & $0,33^{* *}$ & $-0,10$ \\
Soz. Unterst. & $-0,08$ & $-0,06$ & $-0,06$ & $-0,09$ & $-0,20^{*}$ \\
Arztbild & $-0,01$ & 0,16 & $-0,27^{*}$ & $-0,15$ & 0,15 \\
\hline
\end{tabular}

Korrelationskoeffizient r nach Spearman ${ }^{* *} \mathrm{p}<.005 ;{ }^{*} \mathrm{p}<.05$

Variablen: Psych. Belast. $=$ Psychosoziale Belastung; Kort. Ang.=Kortisonangst; Einst. Med.=Einstellung gegenüber Medikamenten allgemein; Compl Med.=Compliance bez. Medikamenten allgemein; Compl. Kort.=Compliance bez. Kortison; Soz. Unterst.=Soziale Unterstützung; Arztbild=positives oder negatives Arztbild. Beachte Itempolung: Kortisonangst: hohe Werte= niedrige Angst; alle anderen Variablen: hohe Werte=hohe Merkmalausprägung 
diese Ängste nur in den seltensten Fällen als „irrational“ anzusehen, sondern vielmehr auf erlebte Nebenwirkungen zurückzuführen waren: So zeigten sich die Patienten als „corticophobisch, wenn sie viele Nebenwirkungen der Medikamente am eigenen Leib beobachten konnten“ [11].

Die Ergebnisse sind trotzdem mit Vorsicht zu behandeln, da die Antworten durch Tendenzen einer positiven Selbstdarstellung oder „Erwünschtheit“ wenig Angst, gute Compliance etc. verfälscht sein könnten. Eine weitere mögliche Erklärung für die geringe Kortisonangst ist, dass die in der Regel älteren Patienten meist langjährige Erfahrungen mit Atemwegskrankheiten hinter sich haben und sie sich mit der Notwendigkeit dieser Therapie bereits abgefunden haben. Der Befund, dass nur bei 3,8\% der Patienten von einer starken und bei 12,2\% von einer mittleren Kortisonangst auszugehen ist, steht im Widerspruch zu Zahlen aus der Pädiatrie, wonach bei Kindern/Eltern Kortisonangst wesentlich häufiger vorkommen soll. Leupold (zit. nach [6]) kommt in einer Umfrage an 141 Kinderärzten zu dem Ergebnis, dass bei oraler Applikation in 86,5\% der Fälle eine „Kortikophobie“ vorliege und differenziert diese in „oft auftretend“ (16,9\%), „gelegentlich“ (22,4\%), „selten“ (23,6\%) und „sehr selten“ (17,8\%). Bei inhalativer Anwendung sei diese Angst mit insgesamt 55,6\% geringer ausgeprägt, und zwar „gelegentlich auftretend“ (16,7\%), „selten“ (21,1\%) und „sehr selten“ (17,8\%). Unklar bleibt hier die Frage, wie die Kortisonangst definiert, differenziert und letztlich auch erhoben wurde. Es handelt sich jedoch offenbar um die subjektive Einschätzung von behandelnden Ärzten, was die Aussagekraft der Daten doch sehr einschränkt. Anzumerken ist, dass es sich bei den von uns untersuchten Personen, Rehabilitanden einer Klinik der LVA (Arbeiterrentenversicherung), sicherlich nicht um einen repräsentativen Querschnitt atemwegskranker Menschen in der Bevölkerung insgesamt handelt.

Bei der geringen Kortisonangst könnte es sich um ein schichtspezifisches Problem handeln. So besteht die Möglichkeit, dass Personen aus der Bevölkerungsschicht, aus der sich das Klientel unseres Hauses vorwiegend zusammensetzt, sich weniger mit Informationsquellen, die zu Kortisonangst führen können, auseinandersetzen und Anordnungen von „Autoritäten“ (Ärzte) weniger hinterfragt befolgen als Personen mit höherem Bildungsniveau und/oder anderer Schichtzugehörigkeit. $\mathrm{Zu}$ den u.U. angstauslösenden Informationsquellen gehören z.B. Beipackzettel, auf denen die Nebenwirkungen von Kortisonpräparaten aufgeführt sind oder Darstellungen in den Massenmedien, die häufig ein unsachlich negatives und undifferenziertes Bild der Nebenwirkungen dieser Präparate zeichnen [14]. Das Ausmaß der Kortisonangst zeigte sich allerdings in bisherigen Studien von soziodemographischen Variablen wie Alter, Geschlecht oder Schulbildung als unabhängig $[9,10]$.

Die Korrelationen der Einstellungsbereiche untereinander sowie mit der Kortisonangst liefern zum Großteil Ergebnisse im Sinne der Erwartung. Lediglich der Befund, dass ein positives Arztbild mit schlechter Compliance zusammenhängt, erscheint nicht plausibel und steht im Widerspruch zu Vorbefunden: „der beste Prädiktor für den Umgang mit Medikamenten allgemein ist die Einstellung zu Medikamenten allgemein und das Arztbild. . . der beste Prädiktor für den Umgang mit Kortisonpräparaten ist das Arztbild. . .“ [11]. Eine Erklärung dafür kann nicht gegeben werden.
Die Diskrepanz der Ergebnisse bez. Kortisonangst kann als Hinweis interpretiert werden, dass man durch unterschiedliche Erhebungsmethoden zu ganz unterschiedlichen Ergebnissen gelangen mag. Es könnte sein, dass gerade die vollstandardisierte Erhebung mit Fragebogen (wie bereits oben erwähnt) Antworttendenzen im Sinne einer positiven Selbstdarstellung oder „Erwünschtheit“ fördert.

Wir sind allerdings der Ansicht, dass die Aussagekraft der über objektive Fragebogen erhobenen Daten zur Einschätzung des Ausmaßes von Kortisonängsten höher einzuschätzen ist als die subjektiven Einschätzungen behandelnder Ärzte. Nach unseren Ergebnissen ist zumindest bei dem eher älteren Klientel in der pneumologischen Rehabilitation die Angst vor Kortisonpräparaten geringer als erwartet.

\section{Danksagung}

Wir danken der Bad Reichenhaller Forschungsanstalt für Erkrankungen der Atmungsorgane (Vorstand Prof. Dr. Nolte) für die freundliche Unterstützung.

\section{Literatur}

${ }^{1}$ Cegla H, Berdel D, Fabel H et al. Glukokortikoidtherapie bei obstruktiven Atemwegskrankheiten. Empfehlungen der Deutschen Atemwegsliga. Münch med Wschr 1992; 134, Nr. 50: $825-828$

2 Internationaler Konsensus-Bericht zur Diagnose und Behandlung des Asthma bronchiale. In: Pneumologie. 1993; 47: 245 - 288

${ }^{3}$ Nolte D. Asthma. 5. Aufl. München: Urban \& Schwarzenberg, 1991

${ }^{4}$ Tuft L. „Steroid-Phobia“ in Asthma Management. Annals of Allergy 1979; 42: 152 - 159

${ }^{5}$ Bosse K, Kaiser H, Lamparter-Lang R. Die sogenannte Cortisonangst. Medizinische Klinik 1992; 87: 540 - 543

${ }^{6}$ Cascan Pharma. Kongress-Bericht vom 25-26 März 1993, Palma de Mallorca: Kortikophobie in der Pneumologie - Resignation oder Herausforderung. Wiesbaden: 1993

${ }^{7}$ Geisler L. Leben mit Asthma, Bronchitis, Emphysem. Wiesbaden: Jopp, 1981

${ }^{8}$ Geisler L. Der Asthmakranke. Ein „schwieriger“ Patient? München: Medikon, 1990

${ }^{9}$ Wöller W, Kruse J et al. Psychische Verarbeitung von Cortison bei Patienten mit Asthma bronchiale. Poster auf dem 34. Kongress der Deutschen Gesellschaft für Pneumologie und Tuberkulose. Bochum: 1990.

${ }^{10}$ Wöller W, Kruse J et al. Negatives Kortisonbild bei Patienten mit Asthma bronchiale. Pneumologie 46: 326 - 329

${ }^{11}$ Deuchert F, Petermann F. Vorstudie zur Corticophobie bei Ärzten und Patienten. Unveröfftl. Forschungsbericht. Universität Bremen, oJ

12 Deuchert M, Petermann F, Petro W. Kortison-Angst und ärztliche Intervention. Münch med Wschr 1994; 136, Nr. 3: 29 - 32

${ }^{13}$ Deuchert M, Petermann F, Petro W. Kortison-Angst von Ärzten und Patienten. Münch med Wschr 1996; 138, Nr. 25: 440 - 443

${ }^{14}$ Petermann F, Deuchert M. Die Entstehung von Kortisonängsten. Präv-Rehab 1993; Jahrgang 5, Nr. 3: 109 - 116

${ }^{15}$ Faßbender CF (Hrsg). Arzt, Patient, Zusammenarbeit. Interdisziplinäre Diskussion zur Compliance. 2. Rotenburger Symposion. Studienreihe Boehringer Mannheim 1981

\section{Dr. phil. Albert Hirschbichler}

Klinik Bad Reichenhall der LVA Ndb.-Opf.

Salzburger Str. 8-11

83435 Bad Reichenhall 